\title{
Neuroprotective effect of Ascorbic acid and Ginkgo biloba against Fluoride caused Neurotoxicity
}

\author{
Raghu Jetti ${ }^{1}$, Raghuveer $\mathrm{CV}^{2}$, Mallikarjuna Rao $\mathrm{C}^{3}$, Somayaji $\mathrm{SN}^{1}$, \\ Prakash Babu $\mathrm{B}^{4}$ \\ 1. Department of Anatomy, Melaka Manipal Medical College, Manipal University, Manipal, Karnataka, \\ India. \\ 2. Department of Pathology, Yenepoya Medical College, Yenepoya University, Mangalore, Karnataka, India. \\ 3. Department of Pharmacology, Manipal College of Pharmaceutical Sciences, Manipal University, Manipal, \\ Karnataka, India. \\ 4. Department of Anatomy, Kasturba Medical College, Manipal University, Manipal, Karnataka, India.
}

\begin{abstract}
Excessive consumption of fluoride through drinking water or other sources lead to skeletal and dental fluorosis. According to the world health organization 23 nations are facing the problem of fluorosis. In the recent past researchers describe the non-skeletal fluorosis where soft tissues and major organs are the victims of fluoride toxicity. Hence in the present study we attempted to investigate the structural changes in various brain areas followed by fluoride exposure and to explore the protective effect of ascorbic acid and Ginkgo biloba against fluoride neurotoxicity. Animals were randomly divided into five groups. Control animals received ordinary water; fluoride animals received fluoride water; all the other animals in the study received gum acacia, ascorbic acid and Ginkgo biloba for first 15 days followed by the fluoride water for 30 days. The present experiment showed fluoride caused neurodegeneration in the form of dark, pyknotic neurons could be apoptosis/ necrosis. However the neurodegeneration is less severe in the ascorbic acid and Ginkgo biloba treated animals. Hence the ascorbic acid and Ginkgo biloba may help as alternate therapeutic strategy to treat fluorosis victims.
\end{abstract}

Keywords: Fluorosis, Ascorbic acid, Ginkgo biloba, Hippocampus, Amygdala, Motor cortex, Neurotoxicity

\section{Introduction}

Fluorosis is a global public health problem; prevalent in approximate 23 nation's worldwide [1]. Skeletal and dental fluorosis results from excessive deposition of fluoride in the enamel and bones. Fluoride intoxication also causes damage to soft tissues like liver, kidney, muscle, brain, heart, thyroid, testis, ovary etc $[2,3]$. Fluoride crosses the blood brain barrier causes neurodegeneration [4]. Fluoride accumulates in the neurons and neuroglia showed morphological changes mainly in the hippocampus [5]. Antioxidants and antioxidant rich food supplementation acts as antidote in the fluorosis management and fluoride intoxication [69]. Fluoride exposure causes DNA damage, oxidative stress and several histopathological changes in the neurons [10-12]. There is a positive correlation between the fluoride exposure and oxidative stress in the brain [13]. In vitro and in vivo fluoride exposure resulted oxidative stress in brain, liver, kidney, lung and testes [14]. Fluoride affects the structure and function of the brain, spinal cord and skeletal muscle [15].

The EGb 761 is neuroprotective in various in vitro and in vivo models [16]. In an in vitro study EGb 761 protected the neurons against the hypoxia induced cell death [17] glutamate toxicity [18]. EGb 761 attenuated the verapamil induced neurodegeneration [19]. The EGb 761 showed beneficial effect against toxin induced neural damage such as MPTP, and cyanide [20]. EGb 761 reduced the neuronal damage observed after the transient occlusion of middle cerebral artery in rats [21]. The Ginkgo biloba has neuroprotective role against several neuronal insults [22]. Further Ginkgo biloba plays a role in neuroregeneration [23, 24].

Ascorbic acid is a water-soluble antioxidant present in citrus fruits, vegetables and strawberries [25]. Ascorbic acid is neuroprotective against the sciatic nerve injury in rats [26]. As an antioxidant ascorbic acid attenuates the oxidative cell death [27], apoptosis [28] quenches intracellular reactive oxygen species [29]. Ascorbic acid in several studies showed neuroprotection against ischemia and excitotoxicity [30-32]. Vitamin C protected the post natal rat brain from ethanol induced neurodegeneration [33], and also neuroglial cells from ethanol caused toxic effects [34]. Ascorbic acid attenuated the lead induced apoptosis in hippocampus [35].

Very few experiments addressed the structural changes in the cerebrum caused by the fluoride exposure. With this background the present study was undertaken to investigate the structural changes in the various regions of the brain following fluoride exposure, to investigate the protective effect of ascorbic acid and Ginkgo biloba on the structural changes caused by fluoride exposure. 
Animal care and maintenance:

\section{Materials And Methods}

Adult male wistar rats were used for the experiment; the animals were housed in the central animal house facility of the Manipal University. Animals were placed in polypropylene cages with paddy husk as bedding material at $26 \pm 2^{0} \mathrm{C}$ with two rats together, maintained in standard light and dark cycle. Standard laboratory pellet diet with water ad libitum was provided. All the experiments were carried out according to the Institutional Animal Ethical Committee (IAEC/KMC/21/2009-2010).

\section{Experimental design:}

Adult male wistar rats were divided in to the five groups with six animals in each group.

Group I: Control animals (exposed to $0.5 \mathrm{ppm}$ of fluoride in drinking water)

Group II: Animals received 100 ppm of sodium fluoride via drinking water for 30 days

Group III: Vehicle control animals received $100 \mathrm{mg} / \mathrm{kg}$ body weight of gum acacia for 30 days, followed by $100 \mathrm{ppm}$ of fluoride for 30 days

Group IV: Animals received $100 \mathrm{mg} / \mathrm{kg}$ body weight of ascorbic acid for 15 days followed by $100 \mathrm{ppm}$ of sodium fluoride for 30 days

Group V: Animals received 100mg/kg body weight of Ginkgo biloba for 15 days followed with $100 \mathrm{ppm}$ fluoride water for 30 days.

Administration of Sodium fluoride:

$221 \mathrm{mg}$ of sodium fluoride was dissolved in the $1000 \mathrm{ml}$ of ordinary tap water to achieve the concentration of $100 \mathrm{ppm}$ of fluoride ions. The fluoride water was provided to the animals as ad libitum

\section{Administration of Ginkgo biloba:}

$100 \mathrm{mg}$ of Ginkgo biloba extract was dissolved in $1 \%$ gum acacia solution. This solution was administered to the animals at the $100 \mathrm{mg} / \mathrm{kg}$ body weight with the help of oral gavage needle attached to a syringe.

\section{Administration of ascorbic acid:}

Since Vitamin C is water-soluble, 200mg of ascorbic acid was dissolved in the $20 \mathrm{ml}$ of ordinary tap water, administered to the animals at the dose of $100 \mathrm{mg} / \mathrm{kg}$ body weight with oral feeding needle.

After the experimental period of 45 days the animals were sacrificed with ether anaesthesia, animals were perfused with $4 \%$ paraformaldehyde solution and the brains were collected in $10 \%$ neutral buffer formalin. The brains were processed for histopathological examination, $5 \mu$ thickness coronal sections were taken with the rotary microtome. The sections were mounted on albumin coated slides.

\section{Cresyl violet staining procedure:}

We have followed the method described by the [37] Madhyastha et al., 2002.

\section{Light microscopic examination:}

The stained slides were examined under $10 \mathrm{X}$ and $40 \mathrm{X}$ with light microscope; the CA1, CA3 regions of hippocampus, Amygdala, Motor cortex regions were identified with help of Paxinos and Watson. Proper stained slides without artefacts in the regions of interest were considered for counting the neurons.

\section{Cell counting:}

Ten sections from the each rat for the each area were selected for counting. Number of viable neurons in the CA1, CA3, Amygdala and Motor cortex regions were counted with 400X magnification across $250 \mu$ length with the aid of ocular micrometer. All the slides were coded before the counting to avoid the manual bias. The results were expressed as number of viable cells per unit length of the field (Number of cells/250 $\mu$ ).

\section{Statistical analysis:}

The data were analysed with one way Anova followed by Bonferroni`s post-test using Graph Pad Prism, version 5 (Graph Pad Prism Software inc., USA). The results were expressed as Mean \pm SD, p value less than 0.05 was considered statistically significant.

\section{Results:}

\section{CA1 and CA3 sub regions of hippocampus (CA1 Fig 1, 2), (CA3 Fig 3, 4):}

In the present experiment fluoride caused neurodegeneration in the hippocampus CA1 and CA3 regions. In comparison to the control the mean numbers of viable neurons were less in these areas ( $p$ value $<0.001)$. The numbers of viable neurons were significantly high in the ascorbic acid and Ginkgo biloba treated group's comparison to fluoride group ( $\mathrm{p}$ value $<0.001$ ). 


\section{Amygdala region (Fig 5, 6):}

The number of viable neurons were less in the fluoride group compared to the control (p value <0.001). The number of viable neurons were more in the Ginkgo biloba and ascorbic acid pre-treated groups ( $\mathrm{p}$ value $<0.001)$.

Motor cortex region (Fig 7, 8):

Fluoride received group showed less number of viable neurons compared to control (p value <0.001). Ginkgo biloba and ascorbic acid received groups showed more number of viable neurons compared to the fluoride group.

The results of the present experiment showed marked neurodegeneration in the CA1, CA3 sub regions of hippocampus, amygdala and motor cortex regions of fluoride group. The neurodegeneration is less severe in the ascorbic acid and Ginkgo biloba groups.

\section{Discussion}

Present study results revealed extensive neurodegeneration in the various brain areas exposed to fluoride. Ascorbic acid and Ginkgo biloba received animal brain showed less degeneration. Fluoride exposed brain showed histopathological changes suggesting necrosis/ apoptosis, and these changes are linked to the thyroid hormone levels [36]. The neurodegeneration observed in the present study was similar to histopathological changes observed in this experiment. Another study observed demyelinization, reduction in number of purkinje cells, disappearance of dendrites, mitochondrial swelling, and endoplasmic reticulum dilatation in the brain of fluoride exposed animals [10]. Fluoride and low iodine exposed animal brain showed shrunken, pyknotic, dark stained neurons with small nuclei, spheroid bodies and decrease in cell number of the neurons [37]. The present experiment showed similar changes with the fluoride alone. The investigators of another experiment detected apoptosis of the neurons exposed to the high fluoride and low iodine [38]. The apoptosis observed in fluorosis is confined to the neurons of cortex and hippocampus [39]. Pyramidal cells of hippocampus showed degeneration, atrophy and necrosis, cerebral cortex showed gliosis, chromatolysis and axonal swelling, signs of encephalitis in fluoride animals. The above changes are seen in the present experiment, except the signs of inflammation. They have also noticed congestion of capillaries, areas of haemorrhage [40]. However we could not find the haemorrhage in the cerebrum. Fluoride caused cell membrane involution, mitochondrial swelling, and chromatin clumping in the CA3, CA4, and dentate gyrus regions [41]. Shivarajashankara reported the similar changes in the hippocampus, cerebellum, motor cortex and amygdala; however the amelioration or mitigation of those changes was not considered in that experiment [42]. Neurodegenerative changes are also seen in the hippocampus, cortex, spinal cord and sciatic nerve [43]. Bax and Bcl-2 proteins are involved in fluoride caused apoptosis [44].

Gingko biloba extract reduced the apoptosis observed in several experiments $[45-47,16]$. In an in vitro study the cells pre incubated with EGb 761 decreased hydroxyl ion induced apoptosis [48]. Ginkgolide B one of the constituents of Ginkgo biloba extract promoted cell survival and reduced the spontaneous apoptosis and toxin induced apoptosis [49]. Prenatal exposure to EGb 761 alters the gene expression and promotes cell survival in hippocampus [50]. Ginkgo biloba prevents the excitotoxicity induced cell death offers neuroprotection [51]. Protective effect of Ginkgo biloba is also extended to the neuroglia especially the astrocytes [52]. The antiapoptotic effect of EGb 761 is due to up regulation of Bcl-2/Bax ratio, down regulation of caspase-3 activity [53].

The neuroprotective effect of ascorbic acid is due to its antioxidant activity, treatment with vitamin $\mathrm{C}$ reduced the oxidative stress in chronic fluoride intoxication [54]. Vitamin $\mathrm{C}$ treatment reversed the structural changes caused by the fluoride [55], in our experiment ascorbic acid prevented degenerative changes caused by the fluoride exposure. Ascorbic acid showed antiapoptotic effect in various in vivo and in vitro studies [27, 29, 56]. Ascorbic acid decreases Bax protein, enhances Bcl-2 protein thereby attenuates the apoptosis [35]. Ascorbic acid prevents the free radical caused oxidative damage of the cell membrane [57]. Ascorbic acid also protects the neurons against exitotoxic cell death $[30,31]$. Vitamin $\mathrm{C}$ exerts neuroprotective action through scavenging the oxygen free radicals [58]. A decrease in lipid peroxidation, an increase catalase activities in the hippocampus were the reason for its neuroprotection [59].

\section{Conclusion}

In conclusion we observed neurodegenerative changes in CA1, CA3 region of hippocampus, motor cortex and amygdala of fluoride exposed animals. These changes were less severe in ascorbic acid and Ginkgo biloba received animals. The findings of the present experiment were consistent with the findings of the previous researchers. The natural antioxidants enhances the nutritional status, antioxidant defence of the body thereby decrease the toxicity or the progress of the disease. 


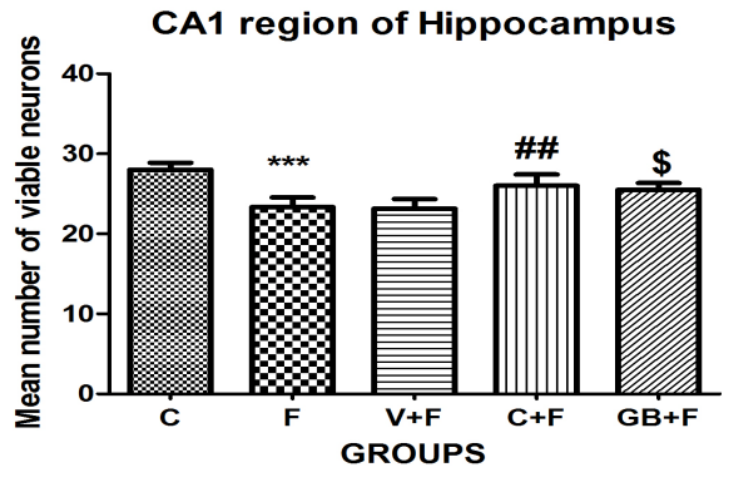

Fig 1: Bar graph shows the number of viable neurons across 250 micron length in CA1 region of hippocampus. C: Control; F: Fluoride, V+F: Vehicle+Fluoride; C+F: Ascorbic acid+Fluoride; GB+F: Ginkgo biloba+Fluoride; $\mathrm{C}$ vs $\mathrm{F}$ p value $<0.001 ; \mathrm{F}$ vs $\mathrm{C}+\mathrm{F}$ p value $<0.01 ; \mathrm{F}+\mathrm{GB}+\mathrm{F}$ p value $<0.05$ one way Anova followed by Bonferroni`s post-test. Each data represents Mean \pm SD.

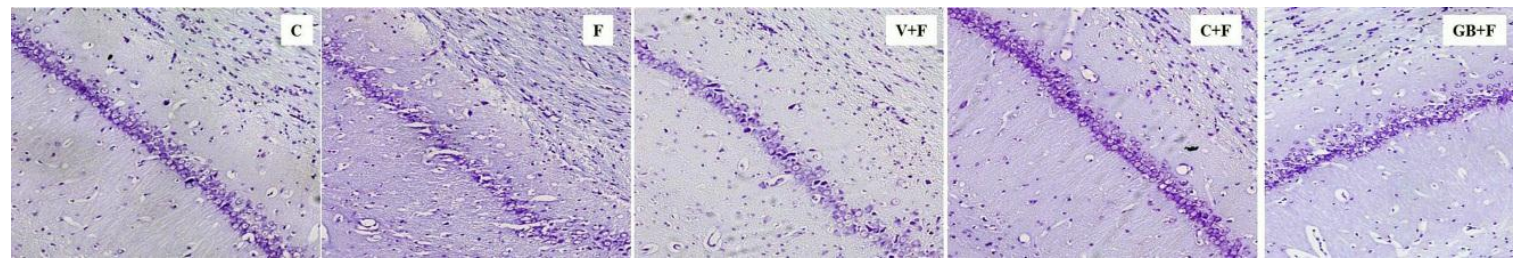

Fig 2: Photomicrograph shows the CA1 region of hippocampus cresyl violet stain (200X). C: Control; F: Fluoride, V+F: Vehicle+Fluoride; C+F: Ascorbic acid+Fluoride; GB+F: Ginkgo biloba+Fluoride.

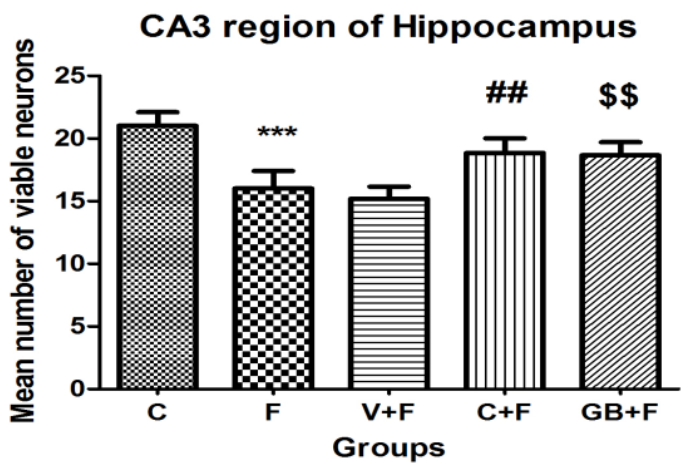

Fig 3: Bar graph shows the number of viable neurons across 250 micron length in CA3 region of hippocampus. C: Control; F: Fluoride, V+F: Vehicle+Fluoride; C+F: Ascorbic acid+Fluoride; GB+F: Ginkgo biloba+Fluoride; $\mathrm{C}$ vs $\mathrm{F}$ p value $<0.001 ; \mathrm{F}$ vs $\mathrm{C}+\mathrm{F}$ p value $<0.01 ; \mathrm{F}+\mathrm{GB}+\mathrm{F}$ p value $<0.01$ one way Anova followed by Bonferroni`s post-test. Each data represents Mean \pm SD.

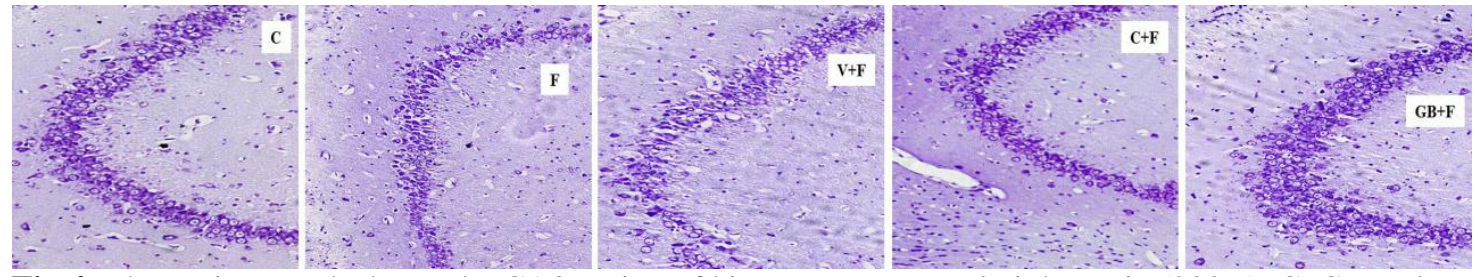

Fig 4: Photomicrograph shows the CA3 region of hippocampus cresyl violet stain (200X). C: Control; F: Fluoride, V+F: Vehicle+Fluoride; C+F: Ascorbic acid+Fluoride; GB+F: Ginkgo biloba+Fluoride. 


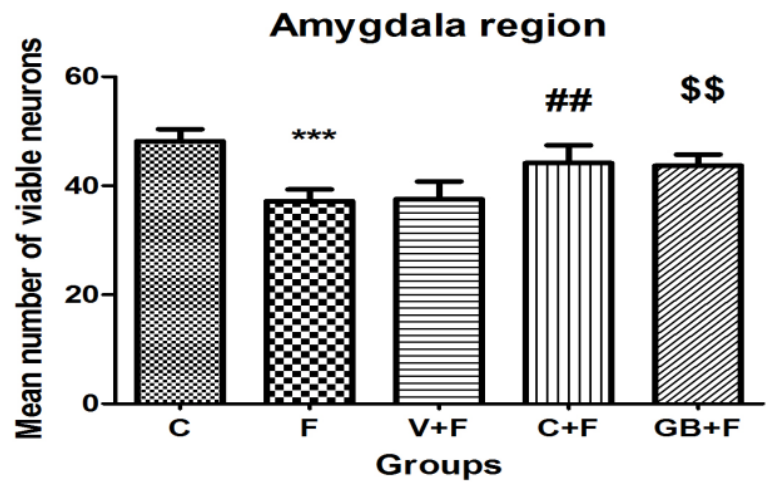

Fig 5: Bar graph shows the number of viable neurons across 250 micron length in amygdala region. C: Control; F: Fluoride, V+F: Vehicle+Fluoride; $\mathrm{C}+\mathrm{F}$ : Ascorbic acid+Fluoride; GB+F: Ginkgo biloba+Fluoride; C vs F p value $<0.001 ; \mathrm{F}$ vs $\mathrm{C}+\mathrm{F}$ p value $<0.01 ; \mathrm{F}+\mathrm{GB}+\mathrm{F}$ p value $<0.01$ one way Anova followed by Bonferroni`s posttest. Each data represents Mean \pm SD.

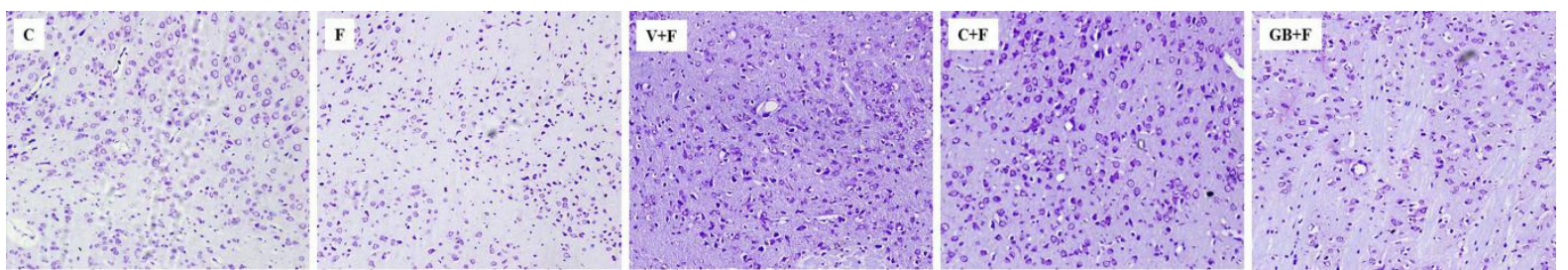

Fig 6: Photomicrograph shows amygdala region cresyl violet stain (200X). C: Control; F: Fluoride, V+F: Vehicle+Fluoride; C+F: Ascorbic acid+Fluoride; GB+F: Ginkgo biloba+Fluoride.

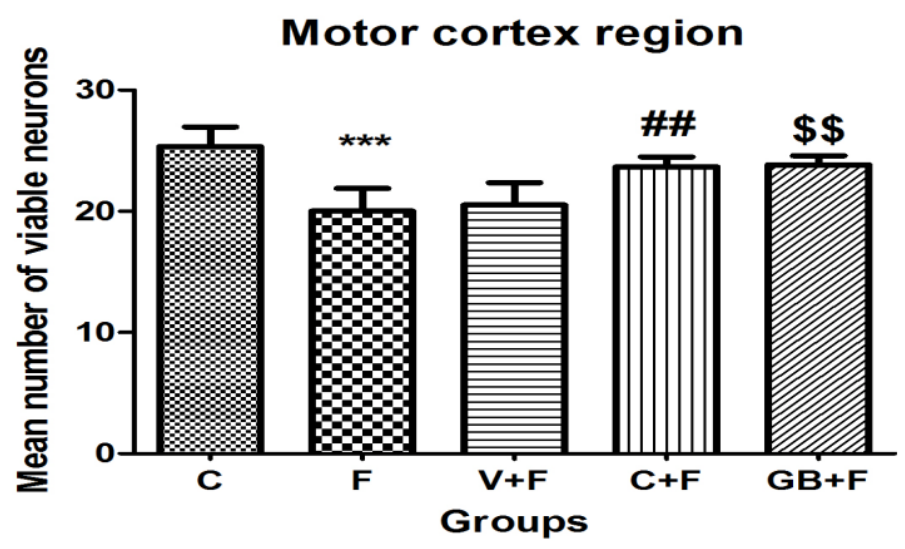

Fig 7: Bar graph shows the number of viable neurons across 250 micron length in motor cortex region. C: Control; F: Fluoride, V+F: Vehicle+Fluoride; C+F: Ascorbic acid+Fluoride; GB+F: Ginkgo biloba+Fluoride; C vs $\mathrm{F} p$ value $<0.001$; F vs $\mathrm{C}+\mathrm{F}$ p value $<0.01 ; \mathrm{F}+\mathrm{GB}+\mathrm{F}$ p value $<0.01$ one way Anova followed by Bonferroni`s post-test. Each data represents Mean \pm SD.

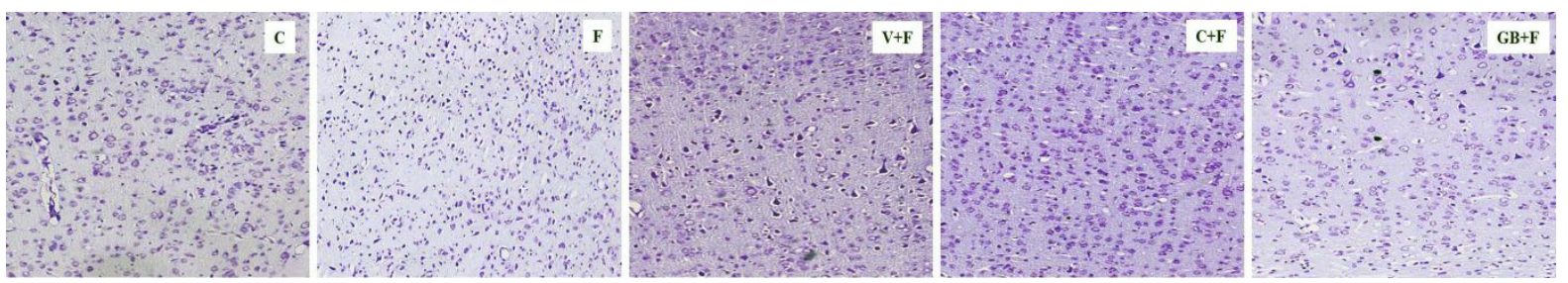

Fig 8: Photomicrograph shows the motor cortex region cresyl violet stain (200X). C: Control; F: Fluoride, V+F: Vehicle+Fluoride; $\mathrm{C}+\mathrm{F}$ : Ascorbic acid+Fluoride; GB+F: Ginkgo biloba+Fluoride. 


\section{Acknowledgements}

We would like to express our gratitude towards the Indian Council of Medical Research for financial assistance (No: 5/8/4-7 (Env) /10-NCD-1.

\section{References}

[1]. AK Susheela, Fluorosis management programme in India, Current Science, 77(10), 1999, 1250-1256.

[2]. K. Chirumari, PK Reddy, Dose-dependent effects of fluoride on neurochemical milieu in the hippocampus and neocortex of rat brain, Fluoride 40(2), 2007, 101-110.

[3]. D. Chlubek, Fluoride and oxidative stress, Fluoride, 36(4), 2003, 217-228.

[4]. YM. Shivarajashankara, AR. Shivashankara, PG. Bhat, SH. Rao, Effect of fluoride intoxication on lipid peroxidation and antioxidant systems in rats, Fluoride, 34(2), 2001, 108-113.

[5]. PJ. Mullenix, PK. Denbesten, A. Schunior, WJ. Kernan, Neurotoxicity of sodium fluoride in rats, Neurotoxicology Teratology, 17(2), 1995, 169-177.

[6]. RJ. Verma, DM. Sherlin. Vitamin C ameliorates fluoride-induced embryotoxicity in pregnant rats, Human and Experimental Toxicology, 20(12), 2001, 619-623.

[7]. AK. Susheela, M. Bhatnagar, Reversal of fluoride induced cell injury through elimination of fluoride and consumption of diet rich in essential nutrients and antioxidants, Molecular and Cellular Biochemistry, 234(1), 2002, 335-340.

[8]. NJ. Chinoy, SD, Shah, Biochemical effects of sodium fluoride and arsenic trioxide toxicity and their reversal in the brain of mice, Fluoride, 37(2), 2004, 80-87.

[9]. MH. Trivedi, RJ. Verma, NJ. Chinoy, RS. Patel, NG, Sathawara, Effect of high fluoride water on intelligence of school children in India, Fluoride, 40(3), 2007, 178-183.

[10]. Y. Ge, H. Ning, S. Wang, J. Wang. Effects of high fluoride and low iodine on brain histopathology in offspring rats, Fluoride, $38(2), 2005,127-132$.

[11]. Y. Ge, H. Ning, S. Wang, J. Wang, Comet assay of DNA damage in brain cells of adult rats exposed to high fluoride and low iodine, Fluoride, 38(3), 2005, 209-214.

[12]. AG. Wang, T. Xia, QL. Chu, M. Zhang, F. Liu, XM. Chen, KD. Yong, Effects of fluoride on lipid peroxidation, DNA damage and apoptosis in human embryo hepatocytes, Biomedical and Environmental Sciences, 17(2), 2004, 217-222.

[13]. SF. Nabavi, S. Eslami, AH. Moghaddam, SM. Nabavi, Protective effects of curcumin against fluoride-induced oxidative stress in the rat brain, Neurophysiology, 43(4), 2001, 287-291.

[14]. O. Barbier, L. Arreola-Mendoza, LM. Del Razo, Molecular mechanisms of fluoride toxicity, Chemico Biological Interactions, 188(2), 2010, 319-323.

[15]. A. Shashi, JP. Singh, SP. Thapar, Protein-degradation in skeletal muscle of rabbit during experimental fluorosis, Fluoride, 25(3), $1992,155-158$.

[16]. B. Ahlemeyer, A. Mowes, J. Krieglstein, Inhibition of serum deprivation-and staurosporine-induced neuronal apoptosis by Ginkgo biloba extract and some of its constituents, European Journal of Pharmacology, 367(2), 1999, 423-430.

[17]. J. Klein, SS. Chatterjee, K. Loffelholz, Phospholipid breakdown and choline release under hypoxic conditions: inhibition by bilobalide, a constituent of Ginkgo biloba, Brain Research, 755(2), 1997, 347-350.

[18]. J. Krieglstein, F. Ausmeier, H. El-Abhar, K. Lippert, M. Welsch, K. Rupalla, Neuroprotective effects of Ginkgo biloba constituents, European journal of pharmaceutical sciences, 3(1), 1995, 39-48.

[19]. L. Zhu, J. Gao, Y. Wang, XN. Zhao, ZX. Zhang, Neuron degeneration induced by verapamil and attenuated by EGb761, Journal of Basic and Clinical Physiology and Pharmacology, 8(4), 1997, 301-314.

[20]. SF. Yang, Q. Wu, AS. Sun, XN. Huang, JS. Shi, Protective effect and mechanism of Ginkgo biloba leaf extracts for Parkinson disease induced by 1-methyl-4-phenyl-1, 2, 3, 6-tetrahydropyridine, Acta Pharmacol Sinica, 22(12), 2001, $1089-1093$.

[21]. WR. Zhang, T. Hayashi, H. Kitagawa, C. Sasaki, K. Sakai, H. Warita, JM. Wang, Y. Shiro, M. Uchida, K Abe, Protective effect of ginkgo extract on rat brain with transient middle cerebral artery occlusion, Neurological Research, 22(5), $2000,517-521$.

[22]. KM. Maclennan, CL. Darlington, PF. Smith, The CNS effects of Ginkgo biloba extracts and ginkgolide B, Progress in Neurobiology, 67(3), 2002, 235-257.

[23]. F. Cheung, YL. Siow, O K, Inhibition by ginkgolides and bilobalide of the production of nitric oxide in macrophages (THP-1) but not in endothelial cells (HUVEC), Biochemical Pharmacology, 61(4), 2001, 503-510.

[24]. A. Didier, D. Rouiller, V. Coronas, F. Jourdan, MT. Droy-Lefaix, Effects of Ginkgo biloba extract (EGb 761) on apoptosis and regeneration of primary olfactory neurons following targetlesioning'in rats, Advances in Ginkgo biloba Extract Research, 5, 1996, 45-52.

[25]. GL. Bowman, Ascorbic acid, cognitive function, and Alzheimer's disease: a current review and future direction, Biofactors, 38(2), 2012, 114-122.

[26]. G. Shokouhi, S. Hadidchi, A. Ghorbanihaghjo, M. Rahbani-Noubar, S. Panahi, M. Forouzanfar N. Rashtchizadeh, M. Mesgariet, Neuroprotective Effect Of Ascorbic Acid In Experimental Blunt Sciatic Nerve Injury In Rats, The Internet Journal of Nutrition and Wellness 1(2), 2005.

[27]. EA. Lutsenko, JM. Carcamo, DW, Golde Vitamin C prevents DNA mutation induced by oxidative stress, Journal of Biological Chemistry, 277(19), 2002, 16895-16899.

[28]. M. Pande, SJS. Flora, Lead induced oxidative damage and its response to combined administration of alpha-lipoic acid and succimers in rats, Toxicology, 177(2), 2002, 187-196

[29]. I Perez-Cruz, JM. Carcamo, DW. Golde, Vitamin C inhibits FAS-induced apoptosis in monocytes and U937 cells, Blood, 102(1), 2003, 336-343

[30]. DG. MacGregor, MJ. Higgins, PA. Jones, WL. Maxwell, MW. Watson, DI. Graham, TW. Stone, Ascorbate attenuates the systemic kainate-induced neurotoxicity in the rat hippocampus, Brain Research, 727(1), 1996, 133-144.

[31]. MD. Majewska, JA. Bell, ED. London, Regulation of the NMDA receptor by redox phenomena: inhibitory role of ascorbate, Brain Research, 537(1), 1990, 328-332.

[32]. JA. Stamford, D. Isaac, CA. Hicks, MA. Ward, DJ. Osborne, MJ. O'Neill, Ascorbic acid is neuroprotective against global ischaemia in striatum but not hippocampus: histological and voltammetric data, Brain Research, 835(2), 1999, $229-240$.

[33]. MI Naseer, U. Najeeb, U. Ikram, H. Zubair Hassan, MBC. Yang, MO. Kim, Vitamin-C protects ethanol induced apoptotic neurodegeneration in postnatal rat brain, Pakistan Journal of Medical Sciences 2, 2009, 718-722.

[34]. C. Sánchez-Moreno, M. Paniagua, A. Madrid, A . Martín, Protective effect of vitamin C against the ethanol mediated toxic effects on human brain glial cells, Journal of Nutritional Biochemistry, 14(10): 2003, 606-613. 
[35]. JM. Han, BJ. Chang, TZ. Li, NH. Choe, FS. Quan, BJ. Jang, IH. Chao, HN. Hong, JH. Lee, Protective effects of ascorbic acid against lead-induced apoptotic neurodegeneration in the developing rat hippocampus in vivo, Brain Research, 1185, $2007,68-74$.

[36]. PM. Basha, P. Rai, S. Begum, Fluoride toxicity and status of serum thyroid hormones, brain histopathology, and learning memory in rats: a multigenerational assessment, Biological and Trace Element Research, 144(1-3), 2011, 1083-1094.

[37]. ZZ. Guan Morphology of the brain of the offspring of rats with chronic fluorosis, Zhonghua Bing Li Xue Za Zhi (Chinese journal of pathology), 15(4), 1986, 297-299.

[38]. Y. Ge, H. Ning, C. Feng, H. Wang, X. Yan, S. Wang, J. Wang, Apoptosis in brain cells of offspring rats exposed to high fluoride and low iodine, Fluoride, 39(3), 2006, 173-178.

[39]. J. Chen, X. Chen, K. Yang, T. Xia, H. Xie Studies on DNA damage and apoptosis in rat brain induced by fluoride, Zhonghua Yu Fang Yi Xue Za Zhi [Chinese journal of preventive medicine], 36(4), 2002, 222-224.

[40]. HS. El-lethey, MM. Kamel, IB. Shaheed, Neurobehavioral toxicity produced by sodium fluoride in drinking water of laboratory rats, Journal of American Science, 6(5), 2010, 54-63.

[41]. M. Bhatnagar, P. Rao, J. Sushma, R. Bhatnagar, Neurotoxicity of fluoride: neurodegeneration in hippocampus of female mice, Indian Journal of Experimental Biology 40(5), 2002, 546-554.

[42]. YM. Shivarajashankara, AR. Shivashankara PG. Bhat, SM. Rao, SH. Rao, Histological changes in the brain of young fluorideintoxicated rats, Fluoride, 35(1), 2002, 12-21.

[43]. PY. Reddy, KP. Reddy, KP. Kumar, Neurodegenerative changes in different regions of brain, spinal cord and sciatic nerve of rats treated with sodium fluoride, Journal of Medical and Allied Sciences, 1(1), 2011, 30-35.

[44]. J. Zhang, Z. Zhang, C. Jinhua, Effects of chronic fluorosis on CAMKIII, C-Fos, BAX and BCL-2 Channel signalling in the hippocampus of rats, Fluoride, 46(3), 2013, 135-141.

[45]. Y. Ni, B. Zhao, J. Hou, W. Xin, Preventive effect of Ginkgo biloba extract on apoptosis in rat cerebellar neuronal cells induced by hydroxyl radicals, Neuroscience Letters, 214(2), 1996, 115-118.

[46]. W. Xin, T. Wei, C. Chen, Y. Ni, B. Zhao, J. Hou, Mechanisms of apoptosis in rat cerebellar granule cells induced by hydroxyl radicals and the effects of EGb761 and its constituents, Toxicology, 148(2), 2000, 103-110.

[47]. C. Guidetti, S. Paracchini, S. Lucchini, M. Cambieri, F. Marzatico, Prevention of neuronal cell damage induced by oxidative stress in-vitro: effect of different Ginkgo biloba extracts, The Journal of Pharmacy and Pharmacology, 53(3), $2001,387-392$.

[48]. C. Chen, T. Wei, Z. Gao, B. Zhao, J. Hou, H. Xu, W. xin, L. Packer, Different effects of the constituents of EGb761 on apoptosis in rat cerebellar granule cells induced by hydroxyl radicals. Biochemistry and Molecular Biology International, 47(3), 1999, 397405 .

[49]. JR. Rapin, M. Zaibi, K. Drieu, In vitro and in vivo effects of an extract of Ginkgo biloba (EGb 761), ginkgolide B, and bilobalide on apoptosis in primary cultures of rat hippocampal neurons, Drug development research, 45(1), 1998, 23-29.

[50]. W. Li, F. Trovero, J. Cordier, Y. Wang, K. Drieu, V. Papadopoulos, Prenatal exposure of rats to Ginkgo biloba extract (EGb 761) increases neuronal survival/growth and alters gene expression in the developing fetal hippocampus, Brain Research. Developmental Brain Research, 144(2), 2003, 169-180.

[51]. K. Chandrasekaran, Z. Mehrabian, B. Spinnewyn, C. Chinopoulos, K. Drieu, G. Fiskum Neuroprotective effects of bilobalide, a component of Ginkgo biloba extract (EGb 761) in global brain ischemia and in excitotoxicity-induced neuronal death, Pharmacopsychiatry, 36(S 1), 2003, 89-94.

[52]. M. Jahanshahi, E. Nikmahzar, N. Yadollahi, K. Ramazani, Protective effects of Ginkgo biloba extract (EGB 761) on astrocytes of rat hippocampus after exposure with scopolamine, Anatomy and Cell Biology, 45(2), 2012, 92-96.

[53]. C. Zhang, C. Ren, H. Chen, R. Geng, H. Fan, H. Zhao, K. Guo, D. Geng. The analog of Ginkgo biloba extract 761 is a protective factor of cognitive impairment induced by chronic fluorosis, Biological Trace Element Research 153(1-3), 2013, $229-236$.

[54]. V. Ailani, RC. Gupta, SK. Gupta, K. Gupta, Oxidative stress in cases of chronic fluoride intoxication, Indian Journal of Clinical Biochemistry, 24(4), 2009, 426-429.

[55]. SD. Shah, NJ. Chinoy, Adverse effects of fluoride and/or arsenic on the cerebral hemisphere of mice and recovery by some antidotes, Fluoride, 37(3), 2004, 162-171

[56]. AM. Sharifi, S. Baniasadi, M. Jorjani, F. Rahimi, M. Bakhshayesh, Investigation of acute lead poisoning on apoptosis in rat hippocampus in vivo, Neuroscience Letters 329(1), 2002, 45-48.

[57]. B. Frei, On the role of vitamin $\mathrm{C}$ and other antioxidants in atherogenesis and vascular dysfunction, Proceedings of the Society for Experimental Biology and Medicine, 222(3), 1999, 196-204.

[58]. Y. Peng, KH. Kwok, PH. Yang, SS. Ng, J. Liu, OG. Wong, ML. He, HF Kung, MC. Lin, Ascorbic acid inhibits ROS production, NF-kB activation and prevents ethanol-induced growth retardation and microencephaly, Neuropharmacology, 48, $2005,426-434$.

[59]. LF. Santos, RL. Freitas, SM. Xavier, GB. Saldanha, RM. Freitas. Neuroprotective actions of vitamin C related to decreased lipid peroxidation and increased catalase activity in adult rats after pilocarpine-induced seizures, Pharmacology Biochemistry and Behavior, 89(1), 2008, 1-5. 